\title{
Aortic Valve replacement via Right Anterior mini-Thoracotomy: a simplified approach to be less traumatic
}

\author{
Gabriele Tamagnini ${ }^{1}$, Raoul Biondi ${ }^{1}$, Jose Zulueta ${ }^{1}$, and Mauro Del Giglio ${ }^{1}$ \\ ${ }^{1}$ Villa Torri Hospital
}

July 1, 2020

\begin{abstract}
Minimally Invasive Aortic Valve Replacement is not just a metric of the incision, but rather a holistic approach to minimize the surgical trauma: our technique tries to reproduce the gold-standard conventional procedure in terms of safety, effectiveness and operative times through a small and different incision. Thanks to a thorough optimization, the surgical times can be at least equal to the classic approach. We started this technique about 10 years ago and after more than 1000 of aortic valve replacement via right anterior mini-thoracotomy, we came up with some tips\&tricks to make this technique our approach of choice.
\end{abstract}

\section{Central Message}

Minimally Invasive AVR (Mi-AVR) should minimize the surgical trauma to reproduce the gold-standard conventional procedure in terms of safety, effectiveness and operative times through a different approach.

\section{Article}

Minimally Invasive aortic valve replacement has gained consent[1] because of minimized surgical trauma, faster rehabilitation, pain control and patient compliance. We try to replicate the conventional and goldstandard approach through a smaller incision[2], [3]. Sparing the right mammary artery, avoiding rib fractures, performing total central cannulation[4] - pivotal to improve perfusion and drainage - and short operative times are important to make that procedure minimally invasive from a biological point of view.

After more than 1000 RAT-Aortic Valve Replacement, we optimized our technique (Central Picture). The patient lies in supine decubitus, with an inflatable bag behind the right scapula to tilt the thorax toward the left side. Double lumen intubation is provided to allow the exclusion of the right lung. A 5-cm skin incision is done and the second or third right intercostal space is opened, respecting the right mammary artery and avoiding rib damage. To identify the correct site of the thoracotomy, we look for the angle of Louis and then the third rib: we incise along its edge for $5 \mathrm{~cm}$, starting $5 \mathrm{~cm}$ far from the jugular-xiphoid line (Fig. 1). Usually the third intercostal space (just below the incision line) is the proper space to open: the right superior pulmonary vein should be in the center of the surgical field. However, it's easy to change space and go to the upper one from the same incision, if the anatomical variance would offer a poor exposition. A soft tissue retractor and a spreader are used to help to spread the intercostal space. The pericardium is opened as far as possible from the phrenic nerve, almost as in median sternotomy. That incision will run-up to the pericardial reflection and down towards the diaphragm, to expose as much as possible the right atrium. We use three pericardial retractions, making a deep figure-of-eight stitch with a strong and thick wire: the first at the level of the right superior pulmonary vein, the second as cranial as possible and the last as low as possible; those stitches are passed through the thoracic wall with an EndoClose to optimize the exposure: that allows to bring towards the thoracotomy the aortic root, especially in case of deep and left-sided position (Fig. 2). Our technique strictly relies on the total central direct arterial and venous 
cannulation. The use of central cannulation reduces surgical trauma and avoids potential complications due to the femoral cannulation, allowing a more physiological perfusion flow and better venous drainage. Furthermore, the retrograde arterial flow has been reported to correlate with dissection of the femoral vessel and the aorta, ischemia of the ipsilateral limb, cerebrovascular and renal events. For arterial cannulation, an EOPA cannula is inserted through a double purse-string suture at the pericardial reflection on the aorta (Fig. 3); a three-stage 29-Fr cannula is placed in the right atrium. To cannulate safely the aorta is crucial gaining an optimal view and control of the target spot: a useful trick is pushing down and towards you the aorta with sponge and forceps. As it might be difficult to engage the inferior cava vein with a straight cannula, we use a metal spindle (e.g. the one included in the intracavitary venting small cannula) to give a rounded shape to the venous cannula (Fig. 4). Moreover, we use a snare strongly tied around the venous cannula and the tourniquet next to the right auricula, then we pass that through the thoracic wall to pull the right atrium away and gain a better aortic root exposure. The left ventricle is vented thanks to a small cannula inserted through the right upper pulmonary vein. Continuous carbon dioxide insufflation is used during the operation at a flow of $3 \mathrm{~L} / \mathrm{min}$. The aorta is cross-clamped at the origin of the innominate artery with a Chitwood-DeBakey clamp, without freeing-up the aorta from the pulmonary artery. To achieve a less-crowded operative field, the clamp is inserted through a separate less-than-1-cm skin incision (Fig. 5), just below the lateral part of the clavicula, and is placed following the transverse sinus: we use the suction cannula to make enough space and to avoid any injury. Blood cold cardioplegia is administered in an antegrade fashion. We use a cold blood-based St. Thomas solution cardioplegia enriched with procaine, that allows about $40 \mathrm{~min}$ of ischemia time. Usually we have no need of a second dose: the sutureless bioprosthesis option or the running suture technique allow to save time. In case of significant aortic regurgitation we give a first shot of cardioplegia to arrest the heart and we renew that straight in the ostia: the left main stem is easily reachable and the right ostium could be find with a small dentist looking glass; in the same way we give a second dose of cardioplegia, when needed. A transverse incision of the ascending aorta is performed (Fig. 6), and the valve is excised en bloc. We implant either sutureless or sutured prostheses. In sutured prostheses, the technique of choice is to use three running sutures of 2-0 prolene $(120 \mathrm{~cm})$ with $3 / 4$ big steps each sinus: in our experience, we have almost better hemodynamic performance, a faster implant and none paravalvular leakage. To stretch properly the sutures, we use a silk thread in the middle of the suture for counter traction. The de-airing process is done by a gentle filling of the heart. The ascending aorta incision is closed with a single or double $4 / 0$ prolene suture. The aortic clamp is removed and the patient is weaned

from cardiopulmonary bypass. Ventricular pacing wires are placed on the right ventricle. Hemostasis is crucial when working in a narrow field, hence we use PTFE pledgets on every purse-string.

\section{References}

[1] J. Lamelas and T. C. Nguyen, "Minimally Invasive Valve Surgery: When Less Is More.," Semin Thorac Cardiovasc Surg, vol. 27, no. 1, pp. 49-56, 2015.

[2] E. Mikus, S. Calvi, M. Panzavolta, J. Luis Zulueta, L. Dozza, A. Cavallucci, and M. Del Giglio, "Right Anterior Mini-Thoracotomy: A 'New Gold Standard' for Aortic Valve Replacement?," J. Heart Valve Dis. , vol. 24, no. 6, pp. 693-698, Nov. 2015.

[3] E. Mikus, S. Turci, S. Calvi, M. Ricci, L. Dozza, and M. Del Giglio, "Aortic valve replacement through right mini-thoracotomy: is it really biologically minimally invasive?," The Annals of Thoracic Surgery, vol. 99, no. 3, pp. 826-830, Mar. 2015.

[4] K. Bedeir, M. Reardon, M. Ramchandani, K. Singh, and B. Ramlawi, "Elevated Stroke Risk Associated With Femoral Artery Cannulation During Mitral Valve Surgery.," Semin Thorac Cardiovasc Surg, vol. 27, no. 2, pp. 97-103, 2015.

Central Picture: Our usual approach: Right Anterior mini-Thoracotomy and total central cannulation.

Fig. 1: Site selection for the thoracotomy

Fig. 2: The configuration of the three pericardial stitches to optimize the exposure 
Fig. 3: The direct arterial cannulation

Fig. 4: The rounded shaped venous cannula to engage easier the inferior cava vein

Fig. 5: The cross-clamp manoeuvre: a separate skin incision gives a less crowded surgical field

Fig. 6: The native aortic valve from the surgeon's point of view
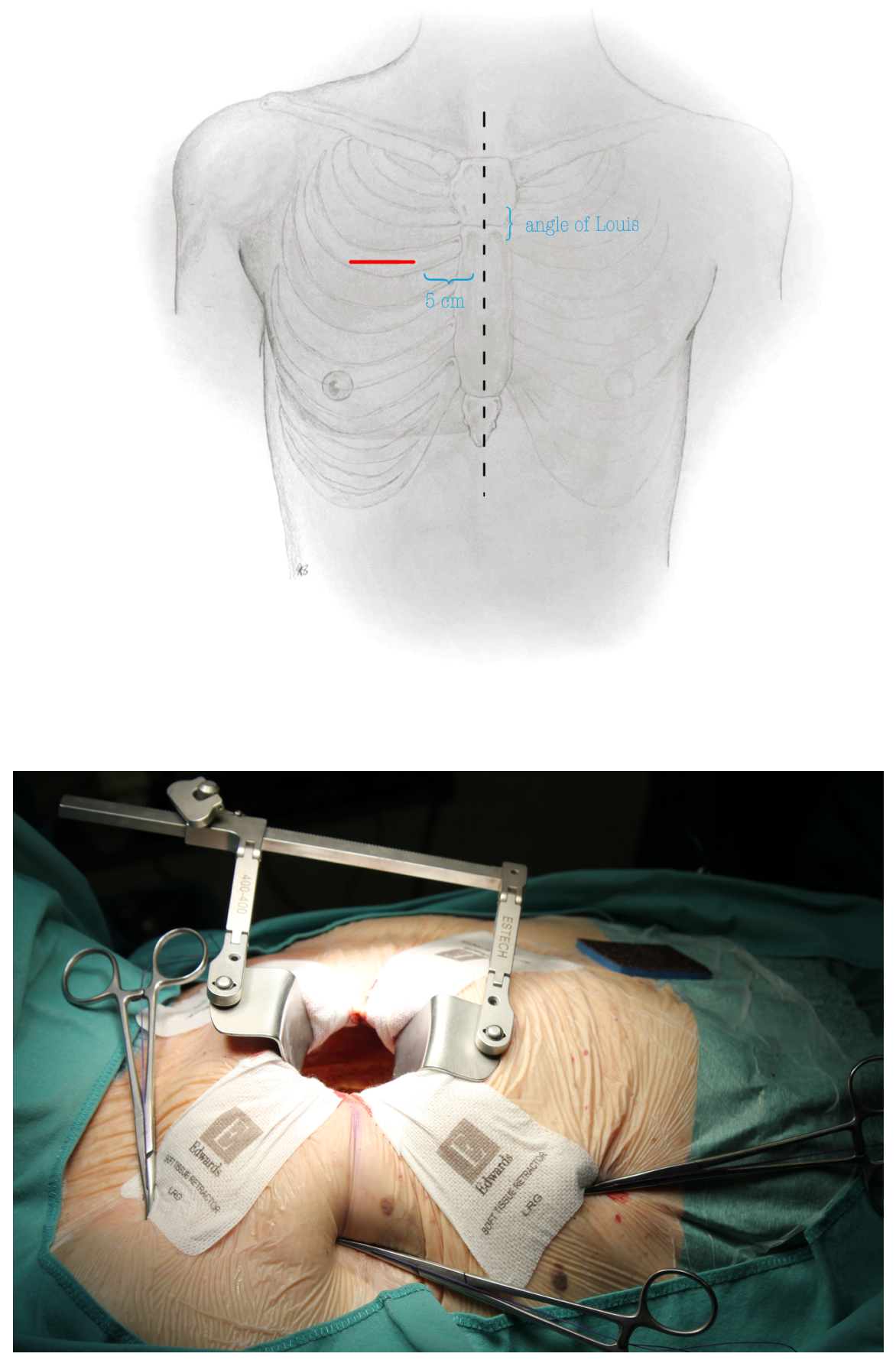

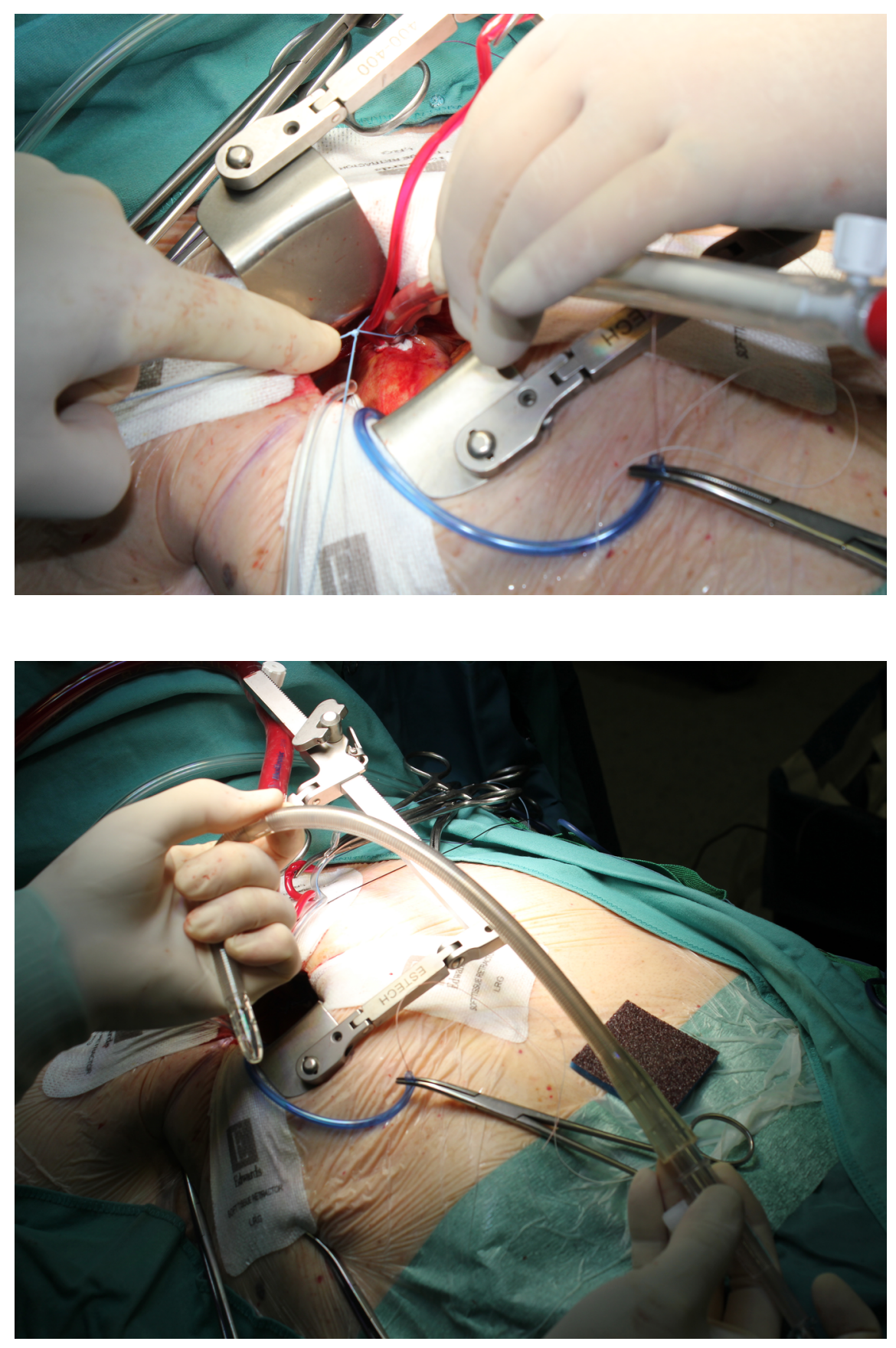


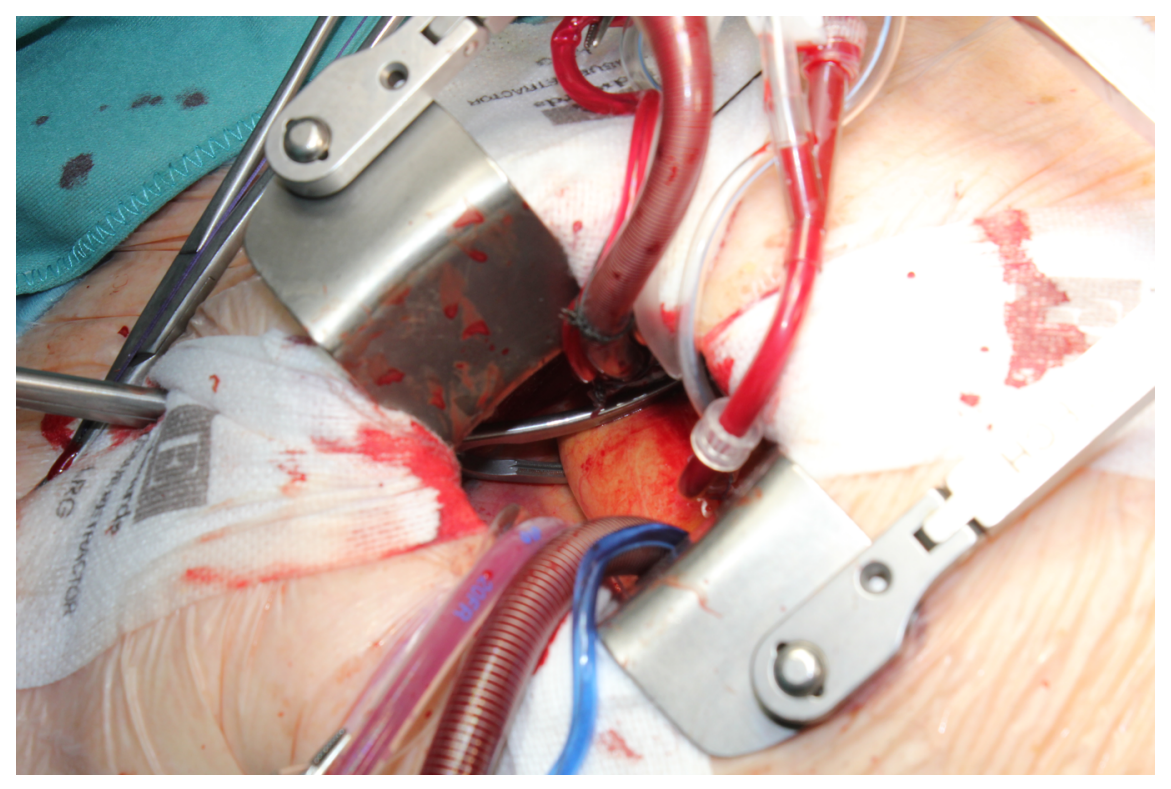




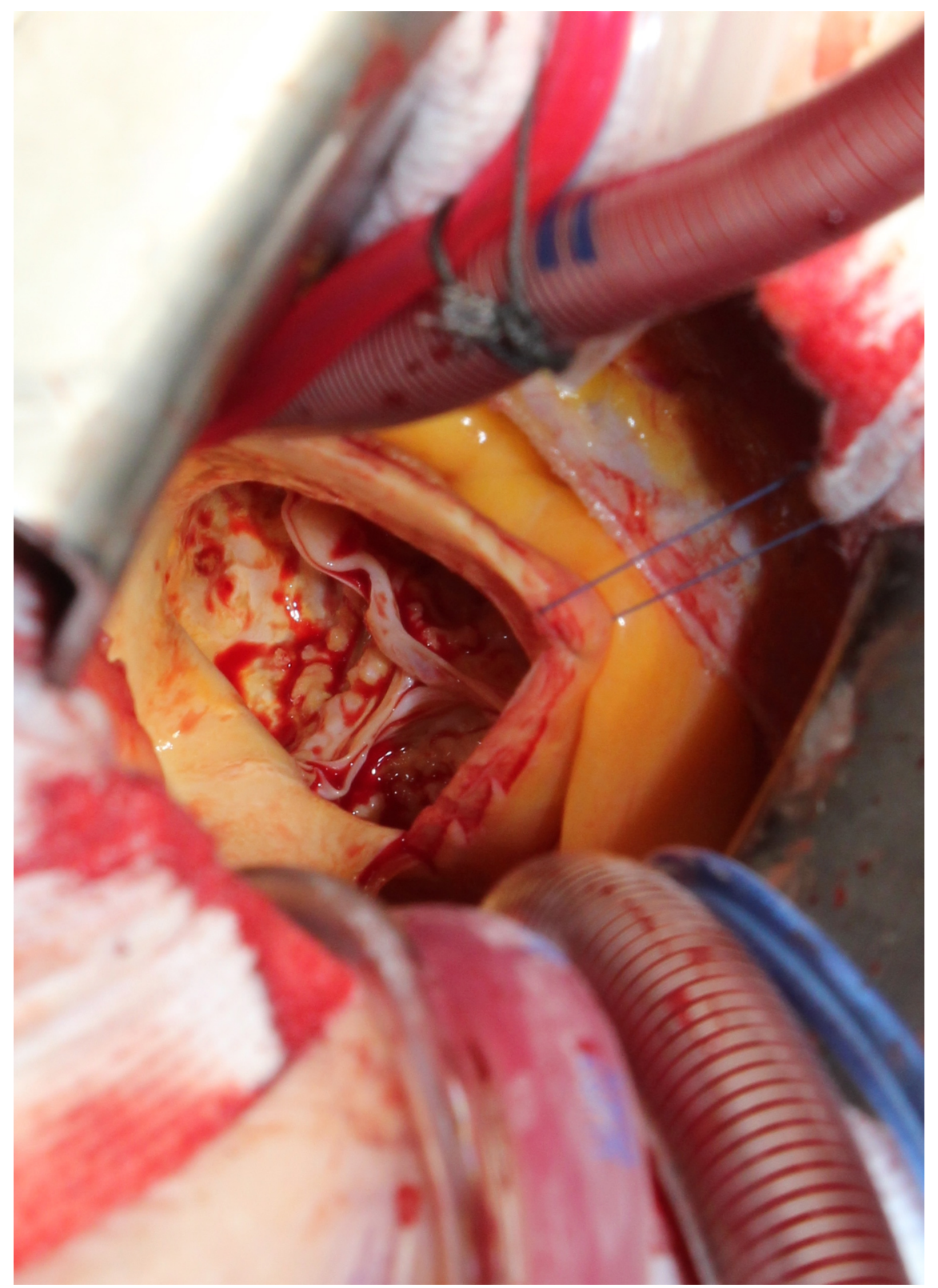




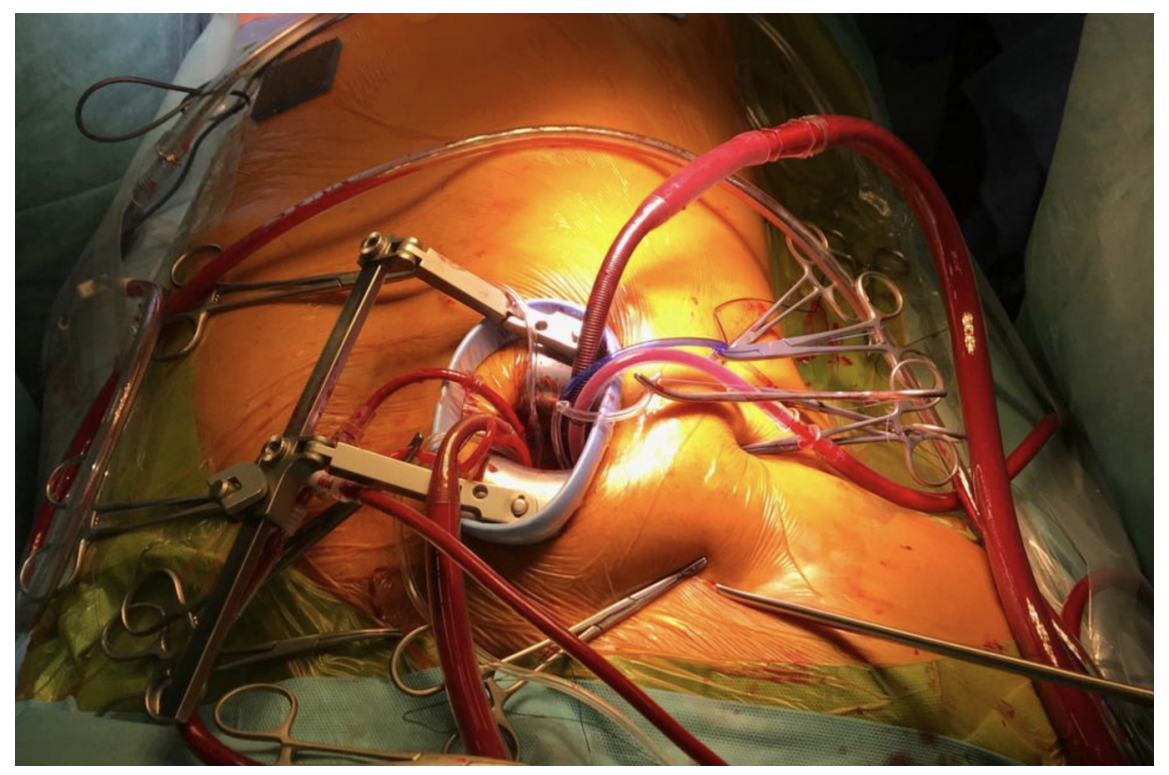

